\title{
Label-free quantitative proteomic analysis of insect larval and metamorphic molts
}

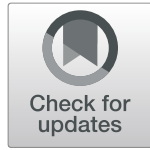

Weiye $\mathrm{Si}^{1 \dagger}$, Qingjie Wang ${ }^{2 \dagger}, \mathrm{Yu} \mathrm{Li}^{1}$ and Dujuan Dong ${ }^{1^{*}}$ (D)

\begin{abstract}
Background: Molting is an essential biological process occurring characteristic times throughout the life cycle of holometabolous insects. However, it is not clear how insects determine the direction of molting to remain status quo or to initiate metamorphosis. To explore the functional factors that determine the direction of molts, liquid chromatography-mass spectrometry was used to identify the molecules involved in larval and metamorphic molting, and the differentially expressed proteins (DEPs) were compared in the two processes.

Results: There were 321 and 1140 DEPs identified in larval and metamorphic molting process, respectively. Bioinformatics analyses show that the amino sugar pathway was up-regulated in both processes. The up-regulated protease contributed to the metamorphosis. In addition, several proteins with different expression patterns in larvallarval and larval-pupal transitions, including Endochitinase, GRIM-19 (Genes associated with retinoid-IFN-induced mortality-19), IDE (Insulin-degrading enzyme), Sorcin (Soluble resistance related calcium binding protein), OBP (Odorant-binding protein-2 precursor), TRAP1(Tumor necrosis factor receptor associated protein-1), etc., were further identified by parallel reaction monitoring, which may play diverse functions in larval-larval and larval-pupal transitions.

Conclusions: These results provide a proteomic insight into molecules involved in larval and metamorphic molts, and will likely improve the current understanding of determination of direction of molts.
\end{abstract}

Keywords: Molting, Metamorphosis, Proteomics, Holometabolous insects

\section{Background}

Insects are a dominant terrestrial group on the Earth with wide distribution and tremendous amount. For the holometabolous class of insects, metamorphosis produced distinct morphology between larva and adult, which enhanced their adaptability to environment, and attributed to the evolutionary success [1]. Characteristic times molts segmented the lives of complete metamorphic insects into three major life stages, including larvae, pupae, and adults. Previous larval molts progress the larva from one instar to the next, during which a

\footnotetext{
* Correspondence: dongdj@sdu.edu.cn

${ }^{+}$Weiye Si and Qing Jie Wang are authors contributed equally to this work. ${ }^{1}$ Shandong Provincial Key Laboratory of Animal Cells and Developmental Biology, School of Life Science, Shandong University, Qingdao 266237, China Full list of author information is available at the end of the article
}

new cuticle secreted by the monolayer of epidermal cells and the old exoskeleton shed [2]. At the end of final larval instar, metamorphic molts switch the molting direction to pupa to adult. In addition to the replacement of old and new cuticle similar to the larval molts, degradation of larval tissues and remodeling of adult tissues occurred during the metamorphic molts [3-5]. How insects determine the direction of molts, to maintain the status quo or to enter metamorphosis, is one of the most frequently stated problems in research about insect's physiology and development.

Existing researches on insect hormones have provided important information on the mechanism regulating molting direction. 20-hydroxyecdysone (20E) evokes the molts through signaling cascade, while the presence or absence of Juvenile hormone $(\mathrm{JH})$ determines whether 
the larvae progress through their instars or formats pupa [6]. In the presence of $\mathrm{JH}, 20 \mathrm{E}$ directs larval molting. However, when larva acquired sufficient nutrients to survive metamorphosis and reached a "critical weight", circulating JH was removed [7]. Then "pupal specifier", such as broad, came up at the time of pupal commitment in response to $20 \mathrm{E}$ and opened up metamorphosis through inducing the transcription factors needed for pupal differentiation [8]. Meanwhile, the biosynthesis and secretion of ecdysone are coordinately regulated by plethora of signaling pathways, including prothoracicotropic hormone, insulin/insulin-like growth factors, neuropeptides, $\mathrm{JH}$ and $20 \mathrm{E}$, transforming growth factor $\beta$, and hedgehog, etc. [9]. These researches have provided a schematic of how hormones regulate the determination of nature of molts. However, not all of the details have been resolved. What other molecules involve in the process of molting and metamorphosis still needs further identification.

A major technological driver in the identification has been the development of high-sensitivity protein mass spectrometry by allowing made the absolute quantification of proteins. Several proteomic studies have led to identify molting related proteins. For example, proteomic analysis was used to investigate the proteins in molting fluids before pupation and eclosion in the silkworm, Bombyx mori [10]. Proteins in the prothoracic glands (PGs) over the final larval stage have also been characterized in $B$. mori to identify the molecular nature and variety of receptors that through the ecdysteroidogenesis [11]. However, large scale investigation on changes in protein abundance of whole body during status quo and metamorphic molts hasn't been reported.

Here, we used Helicoverpa armigera, a holometabolous crop pest that belongs to Lepidoptera, the species with the greatest impacts on agriculture, as a model to explore more molecules involved in larval-larval and larval-pupal transitions.

The proteome of fifth and sixth instar larvae at feeding and molting stages were identified by liquid chromatography-mass spectrometry (LC-MS/MS) respectively. Bioinformatics analysis was used to compare the DEPs which may be involved in larval molting and metamorphic molting. The results show that the upregulated proteins were enriched in the amino sugar metabolism pathway both in larval molting and metamorphic molting, which may be related to the metabolism of chitin involved in the replacement of integument. In addition, the up-regulated proteins in the larval molting are also enriched in the lipid transport process, while the up-regulated proteins in the metamorphic molting stage mainly were associated with protein catabolism. Further, several types DEPs with different expression patterns in larva molting and metamorphosis molting were analyzed by parallel reaction monitoring (PRM) to validate the results of the proteomics. By conducting the research, we may refine the mechanism of determination of direction of molts and find the novel molecular targets to control the pest effectively.

\section{Results}

Larva molting and metamorphic molting in cotton worm

Larva molts segment the life history of cotton worm into six instars, and then the metamorphic molting allows the sixth instar larva transform into the pupae stage (Fig. 1). To identify proteins related to larval molting and metamorphic molting, the feeding and molting stages of fifth and sixth instar larva were sampled for protein preparation and quantitative proteomics analyses respectively.

\section{Identification of differentially expressed proteins}

DEPs occur during different growth and development stages of $H$. armigera. Using mass spectrometry technology, 3386 quantifiable proteins were obtained from the samples. In fifth instar molting stage $(5 \mathrm{M})$ group compared with fifth instar feeding stage (5F) group, there were 321DEPs, of which 161 were up-regulated and 160 were down-regulated (Fig. $2 \mathrm{a}$ and $\mathrm{c}, \mathrm{FC} \geq 2$ or $\mathrm{FC} \leq 0.5$, $P<0.05)$. Compared with sixth instar feeding stage (6F) group, there were 450 up-regulated and 690 downregulated DEPs in sixth metamorphic molting period (6 $\mathrm{M})$ group (Fig. $2 \mathrm{~b}$ and $\mathrm{c}, \mathrm{FC} \geq 2$ or $\mathrm{FC} \leq 0.5, P<0.05$ ). The numbers of 77, 99, 393 and 602 unique proteins were found in the groups of $5 \mathrm{M}$ versus (vs.) $5 \mathrm{~F}$ (up-regulated), $5 \mathrm{M}$ vs. $5 \mathrm{~F}$ (down-regulated), $6 \mathrm{M}$ vs. $6 \mathrm{~F}$ (up-regulated) and $6 \mathrm{M}$ vs. $6 \mathrm{~F}$ (down-regulated), respectively (Fig. 2d). A total of 38, 42, 46, and 19 same proteins were identified in two groups, including the groups of 5 $\mathrm{M}$ vs. $5 \mathrm{~F}$ (up-regulated) and $6 \mathrm{M}$ vs. $6 \mathrm{~F}$ (up-regulated), the groups of $5 \mathrm{M} v s .5 \mathrm{~F}$ (down-regulated) and $6 \mathrm{M} v s$. $6 \mathrm{~F}$ (down-regulated), the groups of $5 \mathrm{M} v s .5 \mathrm{~F}$ (up-regulated) and $6 \mathrm{M} v s .6 \mathrm{~F}$ (down-regulated), and the groups of $5 \mathrm{M} v s .5 \mathrm{~F}$ (down-regulated) and $6 \mathrm{M} v s .6 \mathrm{~F}$ (up-regulated), respectively. However, there were no common elements among all the four groups (Fig. 2d). The detailed information of all identified proteins is shown in Additional file 1.

\section{Gene ontology (GO) enrichment analysis}

To further identify their functions, different categories were performed to annotate all the DEPs. GO is an important bioinformatics analysis tool, which were classified into three categories: biological process (BP), cellular component (CC) and molecular function (MF). After GO enrichment analysis of DEPs in different comparison groups, the correlation of them was compared using the clustering analysis and the results were presented using 


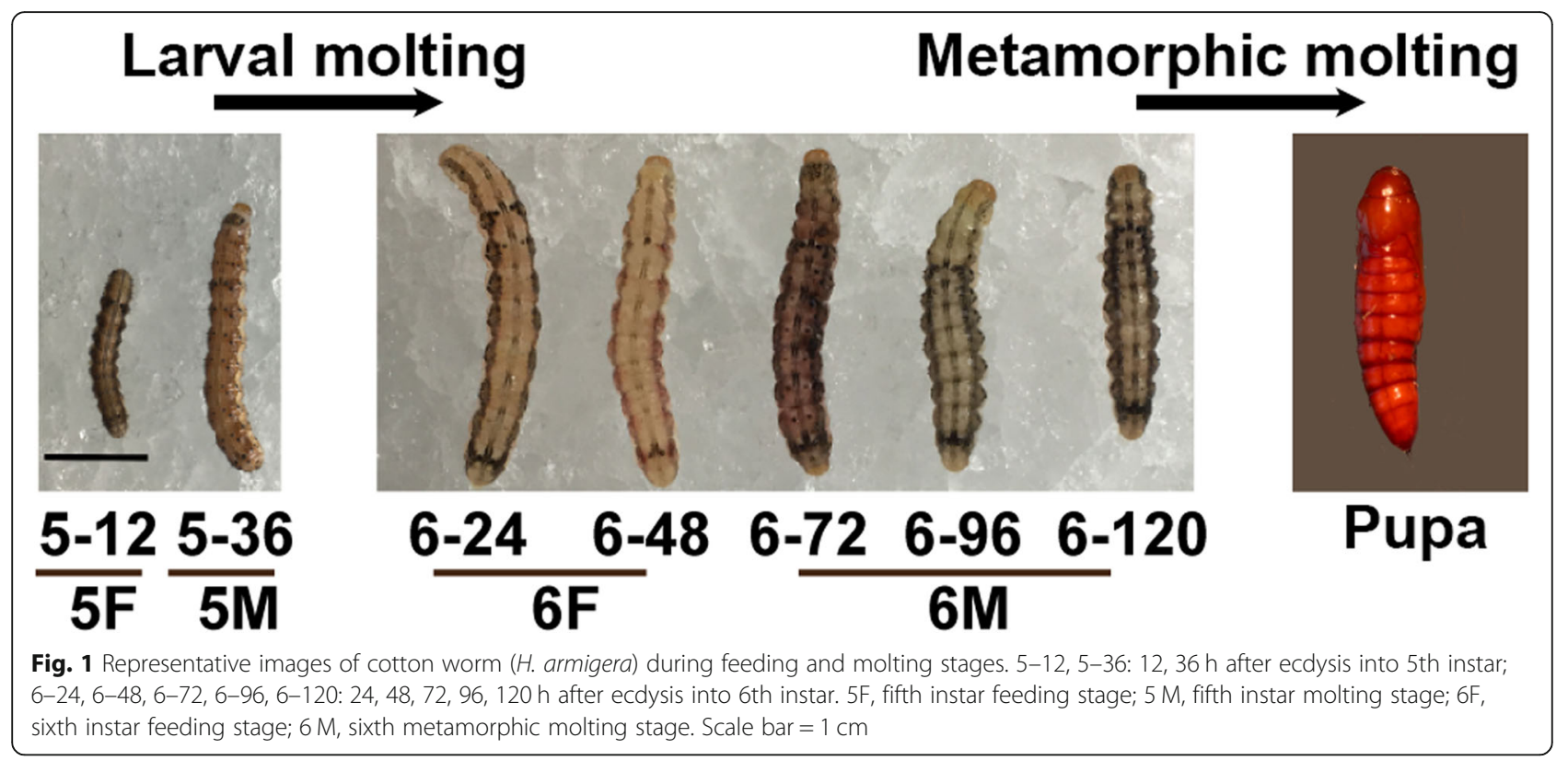

a heat map (Fig. 3). The vertical direction is the description of DEPs enrichment related functions, and the horizontal direction indicates the results of enrichment test of the different groups. Different color blocks indicate the degree of enrichment, and the deeper the red color is, the higher the enrichment degree is. There were significant differences in GO terms level 2 among the four groups.

In BP categories: the up-regulated DEPs from $5 \mathrm{M}$ vs. $5 \mathrm{~F}$ group were associated with terms including lipid transport, glucosamine-containing compound catabolic process, and amino sugar catabolic process. The upregulated DEPs from $6 \mathrm{M}$ vs. $6 \mathrm{~F}$ group were mainly clustered into cellular protein catabolic process, singleorganism carbohydrate catabolic process, proteolysis and protein catabolic process. The remarkable thing is that for up-regulated DEPs, "glucosamine-containing compound metabolic process" and "chitin metabolic process" showed a certain degree of enrichment in both $5 \mathrm{M}$ vs. $5 \mathrm{~F}$ and $6 \mathrm{M}$ vs. $6 \mathrm{~F}$ group (Fig. 3a).

In CC categories: the up-regulated DEPs from $6 \mathrm{M} v s$. 6F group displayed obvious enrichment in extracellular region, proteasome core complex, and alpha-subunit complex. Meanwhile, the down-regulated DEPs from 6 $\mathrm{M}$ vs. $6 \mathrm{~F}$ group were clustered into small ribosomal subunit and large ribosomal subunit (Fig. 3b).

In MF categories: the up-regulated DEPs from $5 \mathrm{M}$ vs. $5 \mathrm{~F}$ group were mainly clustered into chitinase activity, transporter activity and structural constituent of cuticle. The up-regulated DEPs from $6 \mathrm{M}$ vs. $6 \mathrm{~F}$ group were mainly related to some enzyme activities, such as peptidase, endopeptidase, hydrolase, etc. Meanwhile, the down-regulated DEPs from $5 \mathrm{M}$ vs. $5 \mathrm{~F}$ group mainly participated in transferase activity, transferring glycosyl groups and oxidoreductase activity, etc. The downregulated DEPs from $6 \mathrm{M}$ vs. $6 \mathrm{~F}$ group were linked to the translation related activity, such as translation termination factor activity and translation release factor activity, etc. The common functional annotations were found in both larval molting group and metamorphic molting group. The up-regulated DEPs displayed obvious enrichment in chitinase activity, and the downregulated DEPs were clustered into metallopeptidase activity (Fig. 3c).

These results implied that the glucosamine-containing compound metabolic and chitin metabolic may be associated with molting process and the DEPs between larval molting and metamorphosis molting may be the key factors to determine the molting properties.

\section{KEGG (Kyoto encyclopedia of genes and genomes) enrichment analysis}

In order to further understand the pathway information associated with the molting process of the cotton bollworm, KEGG enrichment analysis was performed on the DEPs in each comparison group.

Up-regulated amino sugar and nucleotide sugar metabolism and lysosome pathway was contributed in both $5 \mathrm{M}$ vs. $5 \mathrm{~F}$ and $6 \mathrm{M}$ vs. $6 \mathrm{~F}$ groups (Fig. $4 \mathrm{a}$ ), while there were no common down-regulated pathways in the two groups (Fig. 4c). Importantly, the two groups also contained specific pathways, respectively. Cluster analysis of up-regulated pathways showed that the proteins from "Glycosphingolipid biosynthesis" and "Pyrimidine metabolism" were enriched in $5 \mathrm{M} v s$. $5 \mathrm{~F}$ group, the proteins from "Glycosaminoglycan degradation" and "Insect 


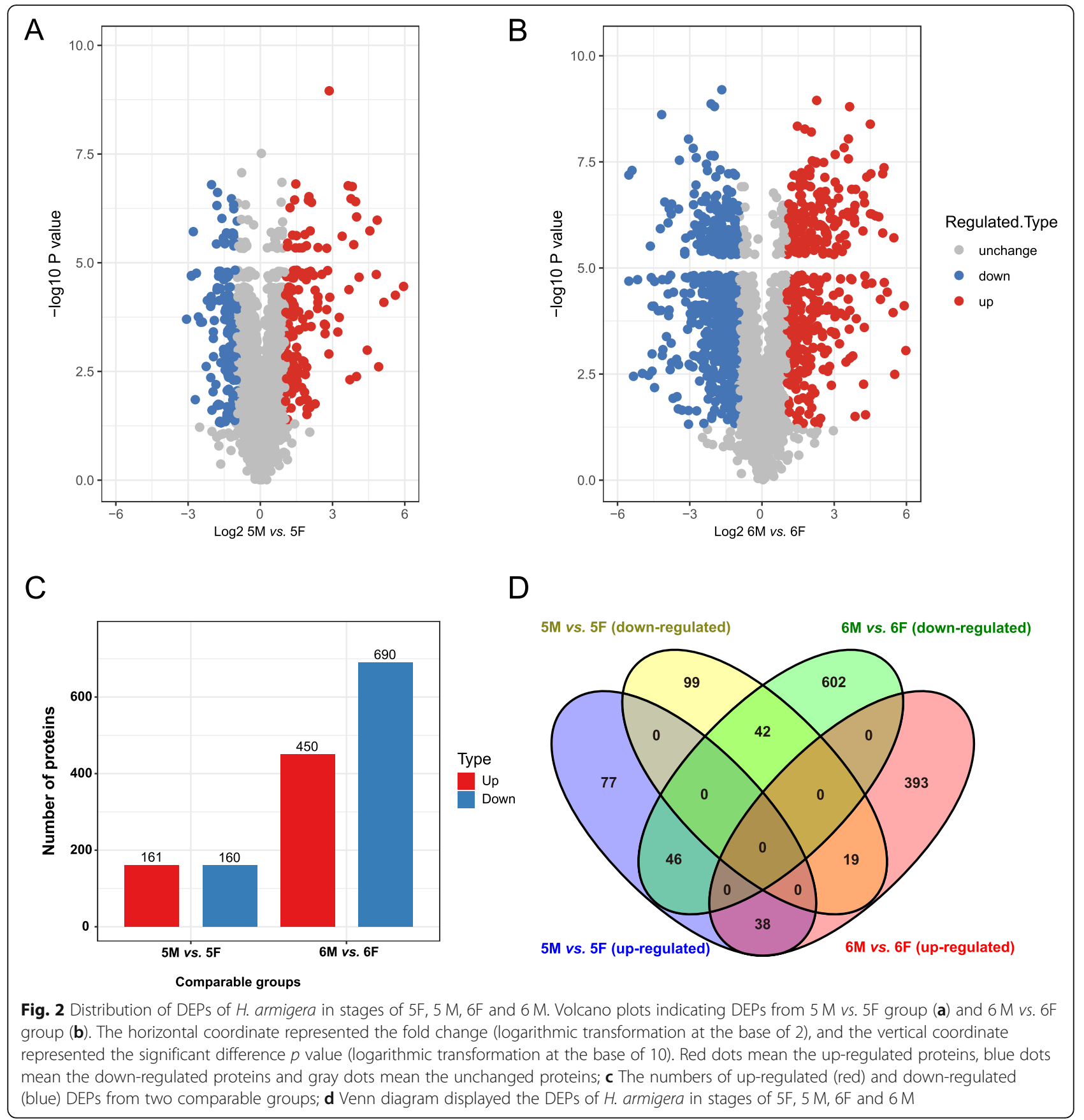

hormone biosynthesis" were enriched in $6 \mathrm{M}$ vs. $6 \mathrm{~F}$ group. In the down-regulated pathway, "Neuroactive ligand-receptor interaction" and "Ribosome" were enriched significantly in $5 \mathrm{M} v s .5 \mathrm{~F}$ group and $6 \mathrm{M} v s .6 \mathrm{~F}$ group respectively. The results showed that there were significant differences in larval molting and metamorphic molting process.

\section{PRM validation analysis}

To confirm the authenticity and accuracy of the proteomic analysis, seven candidates DEPs in larval and metamorphic molts, which have been proved to play important roles in various life processes, were quantified by PRM assay. The results based on PRM supported the shotgun proteomics discovery and revealed that Endochitinase (Precursor), GRIM-19 (Genes associated with retinoid-IFN-induced mortality-19), IDE (Insulindegrading enzyme), Sorcin (Soluble resistance related calcium binding protein), OBP (Odorant-binding protein-2 precursor), TRAP1(Tumor necrosis factor receptor associated protein-1), and BJHSP (Basic juvenile hormone-suppressible protein 1)(Precursor) were altered 


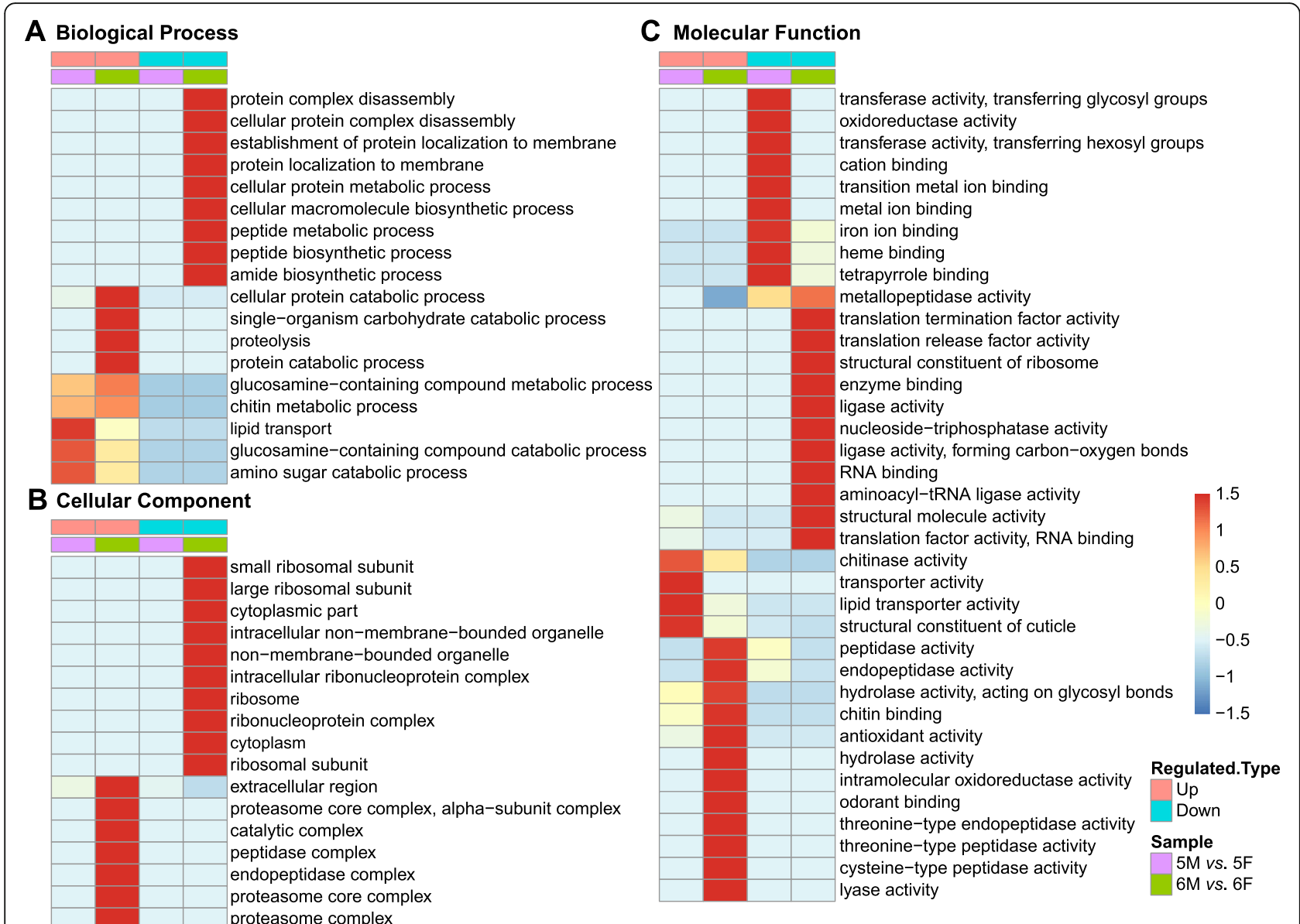

Fig. 3 Cluster analysis heat map based on GO enrichment from $5 \mathrm{M}$ vs. $5 \mathrm{~F}$ group and $6 \mathrm{M}$ vs. $6 \mathrm{~F}$ group. a Biological process; b Cellular component; c Molecular function

expression in different groups (Fig. 5). With reference from the figures, Endochitinase (Precursor) was significantly elevated in both $5 \mathrm{M}$ vs. $5 \mathrm{~F}$ and $6 \mathrm{M} v s$. $6 \mathrm{~F}$ groups $(P<0.05$, respectively). GRIM-19 was significantly decreased in both $5 \mathrm{M}$ vs. $5 \mathrm{~F}$ and $6 \mathrm{M}$ vs. $6 \mathrm{~F}$ groups $(P<0.05$, respectively). IDE was obviously increased in $5 \mathrm{M}$ vs. 5F group and decreased in $6 \mathrm{M}$ vs. $6 \mathrm{~F}$ group, while Sorcin had the opposite change trend $(P<0.05$, respectively). OBP was only downregulated in $5 \mathrm{M} v s$. $5 \mathrm{~F}$ group, while the expression of TRAP1 was only down-regulated in $6 \mathrm{M}$ vs. $6 \mathrm{~F}$ group $(P<0.05$, respectively). The altered expression of BHJSP was only up-regulated in $6 \mathrm{M}$ vs. $6 \mathrm{~F}$ groups $(P<0.05)$.

\section{Discussion}

Using LC-MS/MS and bioinformatics analysis, the specific proteins of fifth and sixth instar larvae at feeding and molting stages were identified in the present study, which provided potential targets to the determination of molting properties and biological control of pest.

\section{Up-regulated amino sugar pathway was contributed in both larval molting and metamorphic molting}

$\mathrm{GO}$ analysis showed that the up-regulated proteins in larval and metamorphosis molting were enriched in the process of "glucosamine-containing compound metabolic" and "chitin metabolic" (Fig. 3a). In addition, KEGG analysis showed that the up-regulated proteins in the two groups were highly enriched in the amino sugar and nucleotide sugar metabolism pathway (Fig. 4a). As shown in KEGG PATHWAY DATABASE (https://www.genome.jp/kegg-bin/show_pathway?map= map00520\&show_description=show), all above the processes were interrelated (Fig. 6). These DEPs mainly include chitinase, $\beta$ - $\mathrm{N}$-acetyl-hexosaminidase, phosphoacetylglucosamine mutase, $\mathrm{N}$-acetylglucosamine-6-phosphate deacetylase (GlcNAc-6P deacetylase), etc.

On the one hand, these enzymes are involved in chitin metabolism. Chitin is a kind of N-acetyl- $\beta$-D- 


\begin{tabular}{|c|c|c|c|}
\hline \multirow[t]{2}{*}{$\mathbf{A}$} & Fold enrichment & B & $-\log 10$ (Fisher's exact test $p$ value) \\
\hline & $\begin{array}{lllll}0 & 5 & 10 & 15 & 20 \\
\end{array}$ & \multirow[b]{2}{*}{ Amino sugar and nucleotide sugar metabolism } & \\
\hline Amino sugar and nucleotide sugar metabolism E & $\square$ & & $\square$ \\
\hline Lysosome & & \multirow{3}{*}{$\begin{array}{r}\text { Glycosphingolipid biosynthesis - globo and isoglobo series } \\
\text { Pyrimidine metabolism }\end{array}$} & I \\
\hline Glycosphingolipid biosynthesis - globo and isoglobo series & & & \\
\hline Pyrimidine metabolism & E & & $\square$ \\
\hline Glycosaminoglycan degradation & & Glycosaminoglycan degradation & \\
\hline Insect hormone biosynthesis & & Insect hormone biosynthesis & \\
\hline Other glycan degradation $E$ & & Other glycan degradation & \\
\hline Histidine metabolism & & Histidine metabolism & $\square$ \\
\hline $\begin{array}{r}\text { Glycolysis / Gluconeogenesis } \\
\text { Proteasome }\end{array}$ & & Glycolysis / Gluconeogenesis & \\
\hline $\begin{array}{c}\text { Proteasome } \\
\text { Arginine and proline metabolism }\end{array}$ & & $\begin{array}{r}\text { Proteasome } \\
\text { Arginine and proline metabolism }\end{array}$ & \\
\hline Pentose phosphate pathway & & Pentose phosphate pathway & $\square$ \\
\hline Glycerolipid metabolism & & Glycerolipid metabolism & \\
\hline Arachidonic acid metabolism & & Arachidonic acid metabolism & $\square$ \\
\hline Longevity regulating pathway - multiple species & & Longevity regulating pathway - multiple species & $\beth$ \\
\hline $\begin{array}{l}\text { Biosynthesis of amino acids } \\
\text { Fructose and mannose metabolism }\end{array}$ & & Biosynthesis of amino acids & \\
\hline $\begin{array}{c}\text { Fructose and mannose metabolism } \\
\text { Glutathione metabolism }\end{array}$ & & $\begin{array}{l}\text { Fructose and mannose metabolism } \\
\text { Glutathione metabolism }\end{array}$ & $=$ \\
\hline $\begin{array}{r}\text { Carbon metabolism } \\
\text { Drug metabolism - cytochrome P450 }\end{array}$ & Up-regulated & $\begin{array}{r}\text { Carbon metabolism } \\
\text { Drug metabolism - cytochrome P450 }\end{array}$ & Up-regulated \\
\hline C & 1,5 & D & $\begin{array}{lll}15 & 20 & 25 \\
\end{array}$ \\
\hline Neuroactive ligand-receptor interactionE- & & Neuroactive ligand-receptor interaction $\square$ & \\
\hline Drug metabolism - cytochrome P450 & & Drug metabolism - cytochrome P450 & \\
\hline Metabolism of xenobiotics by cytochrome & & Metabolism of xenobiotics by cytochrome $=$ & \\
\hline P450 Retinol metabolismE & & P450 Retinol metabolismE & \\
\hline Porphyrin and chlorophyll metabolism & & Porphyrin and chlorophyll metabolism & \\
\hline Drug metabolism - other enzymesE & & Drug metabolism - other enzymes & \\
\hline Ascorbate and aldarate metabolism & & Ascorbate and aldarate metabolism E & \\
\hline Glutathione metabolism & & Glutathione metabolism E & \\
\hline Pentose and glucuronate & & Pentose and glucuronate & \\
\hline interconversions Ribosome- & & interconversions Ribosome $=$ & \\
\hline Aminoacyl-tRNA biosynthesis- & & Aminoacyl-tRNA biosynthesis $\square$ & \\
\hline RNA transport $\square$ & Down-regulated & RNA transport $\square$ & Down-regulated \\
\hline$\square$ 5M vs. $5 \mathrm{~F} \quad \square$ 6M vs. $6 \mathrm{~F}$ & & $\square$ 5M vs. $5 \mathrm{~F} \quad \square 6 \mathrm{M}$ vs. & \\
\hline $\begin{array}{l}\text { Fig. } 4 \text { KEGG enrichment analyses of DEPs from } \\
\text { scores of the up-regulated pathway; } \mathbf{b} \text { was obta } \\
\text { represented the enrichment scores of down-res }\end{array}$ & $\begin{array}{l}\text { n two comparison group } \\
\text { tained using a negative } \\
\text { egulated pathway; } \mathbf{d} \text { was }\end{array}$ & $\begin{array}{l}5 \mathrm{M} \text { vs. } 5 \mathrm{~F} \text { and } 6 \mathrm{M} \text { vs. } 6 \mathrm{~F} \text { in } \mathrm{H} \text {. armigera. a repre } \\
\text { rithm (- } \log 10 \text { Fisher's exact test p value) of up- } \\
\text { ained using a negative logarithm (- } \log 10 \text { Fishe }\end{array}$ & $\begin{array}{l}\text { resented the enrichment } \\
\text {-regulated pathway; } \mathbf{c} \\
\text { er's exact test } p \text { value) }\end{array}$ \\
\hline
\end{tabular}

glucosamine (GlcNAc) linear polymer linked by $\beta-1$, 4glycoside bond, which is the main component of cuticle and midgut membrane of insects. Its synthesis and degradation are very important for the growth and development of insects [12]. Previous studies in Manduca sexta have showed that the synthesis and activation of chitinolytic enzymes, including chitinase and $\beta-\mathrm{N}$-acetyl-hexosaminidase, were increased by ecdysteroid [13]. The $M$. sexta chitinase gene was expressed only before larvallarval, larval-pupal and pupal-adult molting [14]. The LC-MS/MS and PRM analysis of this study showed that Endochitinase (Precursor) was significantly elevated in both $5 \mathrm{M}$ vs. $5 \mathrm{~F}$ and $6 \mathrm{M}$ vs. $6 \mathrm{~F}$ groups, which corroborated the findings of a great deal of the previous work in M. Sexta. Therefore, it seems that both larva molting and metamorphosis molting are involved in chitin metabolism, although they have different directions after molting.

On the other hand, UDP-GlcNAc, a product of hexosamine metabolism, can provide covalent modifying groups for O-GlcNAc modification. Chitinase and GlcNAc-6P deacetylase catalyze the metabolism of hexosamine to the direction of glycolysis, which will reduce
UDP-GlcNAc in cells. This suggests that the decrease of UDP-GlcNAc may be related to the initiation of molting.

\section{Activity of protease is related to metamorphic molting}

In the current study, compared with $5 \mathrm{M}$ vs. $5 \mathrm{~F}$ group, the up-regulated DEPs from $6 \mathrm{M} v s$. $6 \mathrm{~F}$ group, including lysosomal aspartic protease, serine protease, and cysteine proteinase, were mainly clustered into cellular protein catabolic process, single-organism carbohydrate catabolic process, proteolysis and protein catabolic process in BP (Fig. 3a) and peptidase and endopeptidase activity in MF (Fig. 3c). A possible explanation for this might be that the hydrolytic activity of protease is necessary for larval tissue programmed cell death during metamorphosis. In accordance with the present results, prior studies in B. mori have shown that the high expression of cysteine-type cathepsins, aspartic-type cathepsins and metzincins occurs during metamorphic molting. In addition, they participate in the destruction of larval fat body during larval-pupal transition, and they are transcriptionally up-regulated by $20 \mathrm{E}$ signals [15]. 

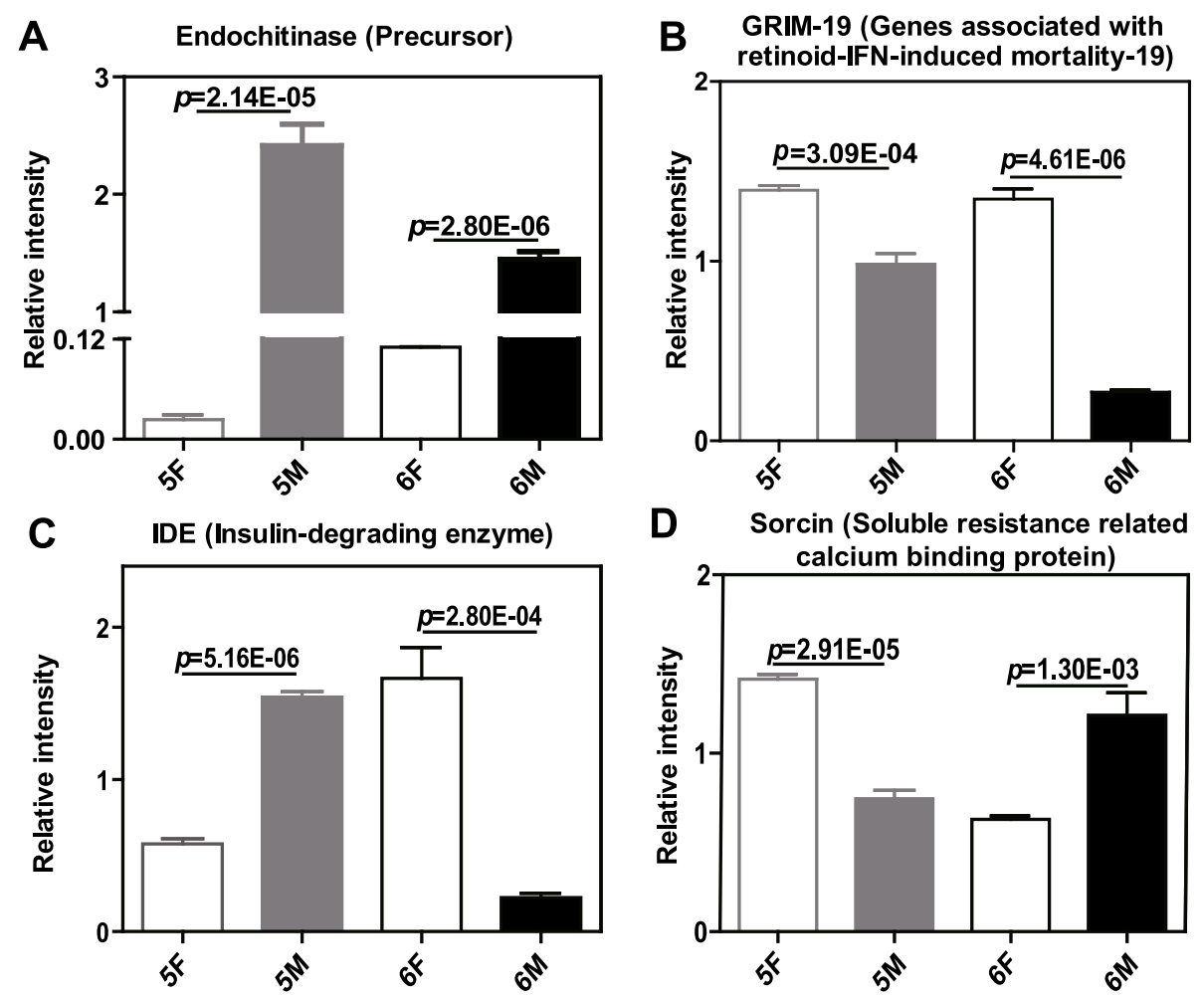

E OBP (Odorant-binding protein-2 precursor)
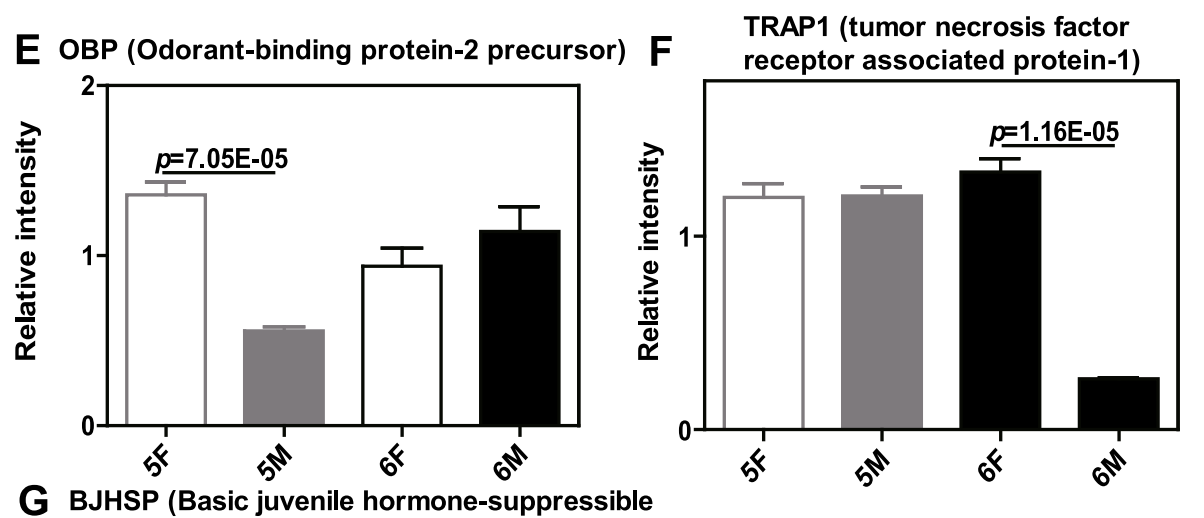

G BJHSP (Basic juvenile hormone-s
protein 1)(Precursor)

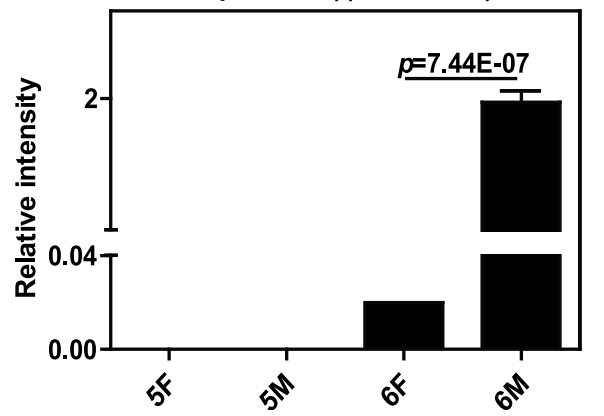

Fig. 5 Using PRM, seven potential DEPs were validated from two comparison groups of $5 \mathrm{M}$ vs. $5 \mathrm{~F}$ and $6 \mathrm{M}$ vs. $6 \mathrm{~F}$ in $\mathrm{H}$. armigera. They showed significant difference between different groups $(P<0.05)$

Data from $H$. armigera have also noted that the cysteine-type cathepsin L participates in midgut apoptosis via caspase-1 [16].
Proteases are widely distributed in organisms. Besides protein hydrolysis, they also have other important physiological functions. Mutations for Carboxypeptidase 


\section{Chitin \\ Chitin synthase \\ Chitinase \\ Chitobiose \\ $\beta-\mathrm{N}$-acetyl- hexosaminidase \\ UDP-GIcNAc \\ UDP-GIcNAc \\ pyrophosphorylase \\ GIcNAc-1P \\ Phosphoacetylglucosamine \\ mutase \\ GIcNAc \\ GICNAc-6P \\ \begin{tabular}{r|r} 
GlcN-6P & GlcNAc-6P \\
acetyltransferase & deacetylase
\end{tabular} \\ GlcN-6P \\ GFAT \\ GlcN-6P \\ isomerase \\ GIc \\ Glc-6P \\ Fru-6P \\ Glycogen Glycolysis \\ synthesis}

Fig. 6 Amino sugar and nucleotide sugar metabolism pathway. The diagram was plotted based on the KEGG pathway database. Glc, Glucose; P, Phosphate; Fru, Fructose; GlcN, Glucosamine; GlcNAc, N-acetylglucosamine

D in Drosophila led to altered wing shape [17] and mating behavior in light [18]. In $H$. armigera, lysosomal aspartic protease cathepsin D promotes midgut apoptosis via the mature form inside cell, and promotes cell proliferation and reassociation of the adult fat body via secreting a pro-enzyme outside the cells as a ligand [19].

One of the issues that emerge from these findings is that up-regulated protease contributed to the initiation of metamorphosis.

The DEPs in larva-larva and larva-pupa transit may be involved in the determination of molting properties In the PRM analysis, we selected seven targets with different trends in the process of larva molting and metamorphosis molting. These targets may help us to find more proteins related to the determination of molting.

Endochitinase (precursor) was significantly elevated in both $5 \mathrm{M}$ vs. $5 \mathrm{~F}$ and $6 \mathrm{M}$ vs. $6 \mathrm{~F}$ groups (Fig. $5 \mathrm{a}$ ). As we discussed above, this result corroborates a great deal of findings in $M$. sexta and $H$. armigera. The $M$. sexta chitinase is abundant during larval-larval, larval-pupal and pupal-adult molting [14]. The $H$. armigera chitinase presents in molting stages but is absent in the intermoult periods [20]. These findings may be due to the fact that chitin is the main component of cuticle, and chitinase is involved in the replacement of old and new epidermis. So molecules up-regulated in both larval-larval and larval-pupal processes in the present study may be involved in the "molting process" itself.

GRIM-19 is a subunit of mitochondrial respiratory complex I. Our previous study showed that GRIM-19 was highly expressed during the larval feeding stage, and the knockdown of GRIM-19 by RNA interference could induced programmed cell death, which imply that GRIM-19 plays roles in keeping the normal cellular growth [21]. Proteomics analysis results that the abundance of GRIM-19 decreased in both $5 \mathrm{M}$ vs. 5F and $6 \mathrm{M} v s .6 \mathrm{~F}$ groups are consistent with our previous observations, which suggested that the proteins enriched in feeding period may keep the normal cellular growth, and inhibit the molting process.

IDE is a zinc metalloprotease that can cleave insulin into inactive fragments. Knocking down IDE in Drosophila can decrease circulating sugar and reduce lifespan, which suggested a role for IDE in determining the level of insulin-like peptides that systemically activate insulin signaling [22]. $20 \mathrm{E}$ and insulin signals have been demonstrated to cross talk with each other to regulate insect development [23]. Insulin signaling pathway promotes larval growth and the ecdysteroid 
production in PGs [24]. 20E, in turn, counteracts insulin pathway to initiate apoptosis and metamorphosis [25]. The antagonism between hormones may be regulated by their titers at different development stages [26]. Our result showed that the expression of IDE was up-regulated in larval molting stage and down regulated in metamorphosis molting stage, which may change the activity of $20 \mathrm{E}$ by changing the concentration of insulin, so as to participate in molting process.

Sorcin is a small soluble penta-EF-family of calcium binding protein [27], which is associated with calcium $\left(\mathrm{Ca}^{2+}\right)$ homeostasis by regulating $\mathrm{Ca}^{2+}$ channels $[28,29]$ or binding with $\mathrm{Ca}^{2+}$ directly [30]. 20E can trigger rapid fluctuation of $\mathrm{Ca}^{2+}$ through membrane receptors and regulate gene expression via a nongenomic signaling pathway [31]. Our result has showed that Sorcin was decreased in $5 \mathrm{M} v s .5 \mathrm{~F}$ group and increased in $6 \mathrm{M} v s .6 \mathrm{~F}$ group, which suggested that Sorcin may play crucial roles in the 20E regulated initiation of metamorphosis through changing $\mathrm{Ca}^{2+}$ level. In addition, sorcin can induce different signaling pathways by modulating the levels of important cellular proteins such as Akt [32], ERK1/2 (Extracellular regulated protein kinases) [33], MMPs (Matrix metalloproteinases) [34], caspases [35], which suggested that Sorcin was associated with the hormonal cross talk in insect development.

OBP, a family of small water soluble proteins containing around 120 to 150 amino acids, plays important role in locating food and mates [36]. The family proteins are able to bind and transport various hydrophobic odorant molecules to the odorant receptors, and then induce the olfactory signal transduction system [37]. In brown planthopper, OBP can be involved in nymph olfaction on rice seedlings, and have non-olfactory functions. Silencing OBP encoding gene resulted in strikingly high nymph mortality, which may be due to disruption of $\mathrm{JH}$ binding and transporting possibly carried out by OBP [38]. Expression patterns analysis showed that OBP has a nymph biased expression and then decreased in the final nymph stage before molting to adults. The present study shows a similar trend. The expression of OBP in feeding stage was higher than that in molting stage, and the altered expression of Odorant-binding protein-2 precursor was only found in $5 \mathrm{M}$ vs. $5 \mathrm{~F}$ group. Our results suggested that OBP may be associated with molts through their binding ability with $\mathrm{JH}$ besides its olfactory functions.

TRAP1 was identified as a novel protein that binds to the receptor for tumor necrosis factor through the yeastbased two hybrid, which shows strong homology to members of the $90-\mathrm{kDa}$ family of heat shock proteins (Hsp90) [39]. Hsp90 are conserved chaperone proteins that protect the structure and function of proteins and play a significant task in cellular homeostasis and signal transduction [40]. In $H$. armigera, Hsp90 contribute to insect development by altering phosphorylation and protein interactions in the cross talk between $20 \mathrm{E}$ and $\mathrm{JH}$ [41]. The result of current study showed that the expression pattern of TRAP1 was in accordance with that of Hsp90 in the fat body of $H$. armigera, which suggests that TRAP1 may have similar function with Hsp90.

BJHSP is a storage protein identified in Trichoplusia $n i$, which can provide the biosynthetic precursors and energy for insect metamorphosis [42]. The mRNA abundance of BJHSP increased dramatically during the metamorphic larval stadium. Maintenance of a high $\mathrm{JH}$ titer caused a strongly suppression for the transcripts of BJHSP [43]. Our proteomic data showed that the expression of BJHSP was not detected in the fifth instar larvae, but only up regulated in the metamorphosis stage of the final instar larvae, which was consistent with the results in $\mathrm{T}$. Ni. These results suggest that after $\mathrm{JH}$ is removed in the metamorphic larval stadium, its inhibition on $\mathrm{BJHSP}$ is released and BJHSP is up-regulated, which may be involved in the initiation of metamorphosis.

\section{Conclusions}

In current study, we identified the functional molecules related to larval molting and metamorphosis molting at proteomic levels, and compared the DEPs in the two processes. The up-regulated amino sugar pathway was detected in both larval molting and metamorphic molting, which indicated that chitin metabolism and fluctuation of O-GlcNAc may be involved in the regulation of molts. The result has also shown that up-regulated protease contributed to the initiation of metamorphosis. In addition, several DEPs with different expression patterns in larva molting and metamorphosis molting, including Endochitinase (precursor), GRIM-19, IDE, Sorcin, OBP, TRAP1, and BJHSP were applied quantitative assays based on PRM. The results supported the shotgun proteomics, and indicated that they may play diverse functions in different mode of molts. Considering the possibility that DEPs may be detected due to normal development of insect, further work needs to be done to establish whether and how the DEPs are relevant to the determination of molting properties.

\section{Methods}

\section{Insects}

The cotton bollworms, $H$. armigera, were bought from Jiyuan Baiyun Industrial Co., Ltd., Henan, China, and were raised in the insectarium at $27^{\circ} \mathrm{C}$ with a $14: 10 \mathrm{~h}$ light-dark cycle. The Larvae were fed on the artificial diet described in previous work [44].

Fifth instar larvae eat for more than $30 \mathrm{~h}$, and the head capsule slippage (HCS) occurred at about 5 th $36 \mathrm{~h}$. Then 
larvae shed the old cuticle and enter 6th instar, the final larval instar. After eating for more than $48 \mathrm{~h}$, 6th larvae begin wandering at $72 \mathrm{~h}$, then the legs contract and pupation at about $140 \mathrm{~h}$. Considering 20E titer pulse [45] and according the phenotype, we prepared the samples at 5th instar feeding (5F, 12-24 h after ecdysis into 5th instar), 5th molting ( $5 \mathrm{M}, 36 \mathrm{~h}$ after ecdysis into 5 th instar), 6th instar feeding (6F, 12-60 h after ecdysis into 6 $\mathrm{h}$ instar), and 6th instar metamorphosis (72-120 h after ecdysis into $6 \mathrm{~h}$ instar) stages.

\section{Protein extraction and trypsin digestion}

Firstly, the whole bodies of bleeding larvae at $5 \mathrm{~F}, 5 \mathrm{M}$, $6 \mathrm{~F}, 6 \mathrm{M}$ stages were grinded in liquid nitrogen. Then the cell powder was sonicated three times on ice in the lysate buffer containing $8 \mathrm{M}$ urea and 1\% Protease Inhibitor Cocktail. After centrifugation $(12,000 \mathrm{~g}$ for $10 \mathrm{~min}$ at $4{ }^{\circ} \mathrm{C}$ ), the supematant was harvested, and the Sigma Bicinchoninic Acid kit was used to measure the remaining protein concentration following the manufacturer's instructions.

After reduction $(5 \mathrm{mM}$ dithiothreitol for $30 \mathrm{~min}$ at $\left.56^{\circ} \mathrm{C}\right)$ and alkylation $(11 \mathrm{mM}$ iodoacetamide in the dark at room temperature for $15 \mathrm{~min}$ ), the protein solution was diluted with $100 \mathrm{mM}$ tetraethylammonium bromide to urea concentration less than $2 \mathrm{M}$. Then the samples were trypsin-digested two times (1:50 trypsin-to-protein overnight and 1:100 for another $4 \mathrm{~h}$ ) and fractionated by high $\mathrm{pH}$ reverse-phase HPLC using a Thermo Betasil C18 column (5 $\mu \mathrm{m}$ particles, $10 \mathrm{~mm}$ ID, $250 \mathrm{~mm}$ length).

\section{LC-MS/MS analysis}

The enriched peptides were loaded onto a $15 \mathrm{~cm}$ length, $75 \mu \mathrm{m}$ i.d. home-made reversed-phase analytical column after being dissolved in solvent A ( $0.1 \%$ formic acid). The gradient of solvent B $(0.1 \%$ formic acid in $98 \%$ acetonitrile) increased from 6 to $23 \%$ in $26 \mathrm{~min}$, from 23 to $35 \%$ in $8 \mathrm{~min}$, and then climbing to $80 \%$ in $3 \mathrm{~min}$ and was held at $80 \%$ for $3 \mathrm{~min}$. All operations were done on an EASY-nLC 1000 UPLC system at a constant flow rate of $400 \mathrm{~nL} / \mathrm{min}$.

After subjected to NSI source, the peptides were analyzed by tandem mass spectrometry (MS/MS) in $\mathrm{Q}$ Exactive $^{\mathrm{Tm}}$ Plus (Thermo) coupled online to the UPLC with $2.0 \mathrm{kV}$ electrospray voltage. For full scan, the $\mathrm{m} / \mathrm{z}$ scan range from 350 to 1800 was used. Intact peptides were detected in the Orbitrap at a resolution of 70,000 and then selected for MS/MS using NCE setting as 28 . The ion fragments were detected in the Orbitrap at a resolution of 17,500. The data-dependent procedure was set as alternation between one MS scan followed by 20 MS/MS scans with $15.0 \mathrm{~s}$ dynamic exclusion. 5E4 was set for automatic gain control and $100 \mathrm{~m} / \mathrm{z}$ was set for fixed first mass.
The mass spectrometry proteomics data have been deposited to the publicly accessible database PRIDE Archive (https://www.ebi.ac.uk/pride/) and can be accessed with the dataset identifier PXD020414.

\section{Database search}

The resulting tandem mass spectra data were queried against our unpublished transcriptome data of $\mathrm{H}$. armigera using the Maxquant search engine (v.1.5.2.8) concatenated with the reverse decoy database. Trypsin/P was specified as cleavage enzyme and up to 4 missing cleavages were allowed. The mass tolerance of $20 \mathrm{ppm}$ was used for precursor ions in first search and $5 \mathrm{ppm}$ in main search, and the mass tolerance of $0.02 \mathrm{Da}$ was set for fragment ions. The fixed modification was carbamidomethyl on Cys and variable modifications contained acetylation modification and oxidation on Met. FDR was adjusted to $<1 \%$ and minimum score for modified peptides was set $>40$.

UniProt-GOA database (http://www.ebi.ac.uk/GOA/) and InterProScan software (http://www.ebi.ac.uk/interpro/, version 5.14-53.0) was used to perform GO annotations. KAAS (http://www.genome.jp/kaas-bin/ kaas_main, version 2.0) and KEGG Mapper (http://www. kegg.jp/kegg/mapper.html, version 2.5) were used to perform KEGG analysis. R Package pheatmap (https://cran. r-project.org/web/packages/cluster/, version 2.0.3) was used to generate the heat map of protein abundance.

\section{Functional enrichment $G O$ enrichment analysis}

According to GO annotation, proteins were classified into three groups based on BP, CC and MF. For each category, the enrichment of differentially abundant proteins relative to all identified proteins was detected using a double-tailed Fisher's precision test. GO with a revised $P$ value of $<0.05$ is considered significant.

\section{Pathway enrichment analysis}

Based on KEGG database, the enriched pathways to test the enrichment of the differentially expressed protein against all identified proteins were performed using a two-tailed Fisher's exact test. The pathway was considered significant with a corrected $p$-value $<0.05$. These pathways were classified into hierarchical categories through the KEGG website.

\section{Enrichment-based clustering}

Further hierarchical clustering were performed based on GO and KEGG. All the categories obtained after enrichment along with their $P$ values were first collated, and followed filtering for those categories which were at least enriched in one of the clusters $(P$ value $<0.05)$. The function $\mathrm{x}=-\log 10$ ( $P$ value) was used to transform the 
filtered $\mathrm{P}$ value matrix and these $\mathrm{x}$ values were $\mathrm{z}$ transformed for each functional category. One-way hierarchical clustering (Euclidean distance, average linkage clustering) in Genesis were used to cluster these $\mathrm{z}$ scores. The "heatmap.2" function from the "gplots" Rpackage was applied to visualize the cluster membership via a heat map.

\section{Parallel reaction monitoring (PRM)}

The tryptic peptides were dissolved in $0.1 \%$ solvent A and injected into an EASY-nLC 1000 UPLC system, using a reversed-phase analytical column $(15 \mathrm{~cm}$ length and $75 \mu \mathrm{m}$ i.d.). The elution gradient was set as $6-23 \%$ solvent B for $38 \mathrm{~min}, 23-35 \%$ for $14 \mathrm{~min}$, climbing to $80 \%$ in $4 \mathrm{~min}$, and finally keeping at $80 \%$ for $4 \mathrm{~min}$.

After subjected to NSI source, the peptides were analyzed by MS/MS in Q Exactive ${ }^{\text {tm }}$ Plus coupled online to the UPLC with $2.0 \mathrm{kV}$ electrospray voltage. A full mass spectrum at 35,000 resolution (AGC target 3E6, $20 \mathrm{~ms}$ maximum injection time, $\mathrm{m} / \mathrm{z} 350-1000)$ was followed by up to 20 PRM scans at 17,500 resolution (AGC target 1E5, auto maximum injection time). PRM data were manually curated within Skyline (v.3.6).

\section{Statistical analysis}

All experiments were performed in triplicate. Column charts and their statistical analysis were made using graph Pad Prism 5.0 software. Statistical comparisons between groups were estimated by Student's t-test and $P<0.05$ was considered statistically significant.

\section{Supplementary Information}

The online version contains supplementary material available at https://doi. org/10.1186/s12861-020-00227-z.

Additional file 1. The detailed information of all identified DEPs in larval and metamorphic molts. The detail information of DEPs in two groups (5 M vs. 5F; $6 \mathrm{M}$ vs. 6F), including protein accession, protein description, gene name, peptide number, matching scores, and functional enrichment analysis results.

\section{Abbreviations}

20E: 20-hydroxyecdyson; DEPs: Differentially expressed proteins; JH: Juvenile hormone; PGs: Prothoracic glands; PRM: Parallel reaction monitoring; LC-MS/ MS: Liquid chromatography-mass spectrometry; 5F: Fifth instar feeding stage 5M: Fifth instar molting stage; 6F: Sixth instar feeding stage; 6M: Sixth instar metamorphic molting stage; GRIM-19: Genes associated with retinoid-IFNinduced mortality-19; IDE: Insulin-degrading enzyme; Sorcin: Soluble resistance related calcium binding protein; OBP: Odorant-binding protein-2 precursor; TRAP1: Tumor necrosis factor receptor associated protein-1; BJHSP: Basic juvenile hormone-suppressible protein 1

\section{Acknowledgements}

Not applicable.

\section{Authors' contributions}

W.S. AND Y. L. conducted the experiments; Q. W. curated and analyzed the data; D.D. designed the experiments and wrote the manuscript. All authors have read and agreed to the published version of the manuscript.

\section{Funding}

The collection, analysis, and interpretation of data were supported by grants from the Natural Science Foundation of China (31672356, 31970464), the Key Research and Development Program of Shandong Province

(2018GSF121026), and Shandong Provincal Key Laboratory of Animal Cell and Developmental Biology (SDKLACDB-2019002).

\section{Availability of data and materials}

The datasets generated or analysed during this study are included in this published article and its supplementary information files.

The raw mass spectrometry proteomics data have been deposited to the publicly accessible database PRIDE Archive (https://www.ebi.ac.uk/pride/) with the dataset identifier PXD020414.

Ethics approval and consent to participate

Not applicable.

\section{Consent for publication}

Not applicable.

\section{Competing interests}

The authors declare that they have no competing interests.

\section{Author details}

${ }^{1}$ Shandong Provincial Key Laboratory of Animal Cells and Developmental Biology, School of Life Science, Shandong University, Qingdao 266237, China. ${ }^{2}$ Laboratory of Basic Medical Sciences, Qilu Hospital, Shandong University, Jinan 250012, China.

Received: 2 September 2020 Accepted: 3 November 2020

Published online: 24 November 2020

\section{References}

1. Yang AS. Modularity, evolvability, and adaptive radiations: a comparison of the hemi- and holometabolous insects. Evol Dev. 2001;3:59-72.

2. Mesce KA, Fahrbach SE. Integration of endocrine signals that regulate insect ecdysis. Front Neuroendocrinol. 2002;23:179-99.

3. Uwo MF, Ui-Tei K, Park P, Takeda M. Replacement of midgut epithelium in the greater wax moth, galleria mellonela, during larval-pupal moult. Cell Tissue Res. 2002;308:319-31.

4. Nelliot A, Bond N, Hoshizaki DK. Fat-body remodeling in Drosophila melanogaster. Genesis. 2006;44:396-400.

5. Li YB, Li XR, Yang T, Wang JX, Zhao XF. The steroid hormone 20hydroxyecdysone promotes switching from autophagy to apoptosis by increasing intracellular calcium levels. Insect Biochem Mol Biol. 2016;79:73-86.

6. Riddiford LM, Ashburner M. Effects of juvenile hormone mimics on larval development and metamorphosis of Drosophila melanogaster. Gen Comp Endocrinol. 1991;82:172-83.

7. Nijhout HF, Williams CM. Control of moulting and metamorphosis in the tobacco hornworm, Manduca sexta (L.): cessation of juvenile hormone secretion as a trigger for pupation. J Exp Biol. 1974;61:493-501.

8. von Kalm L, Crossgrove K, Von Seggern D, Guild GM, Beckendorf SK. The broad-complex directly controls a tissue-specific response to the steroid hormone ecdysone at the onset of Drosophila metamorphosis. EMBO J. 1994;13:3505-16.

9. Pan $\mathrm{XY}, \mathrm{Connacher} \mathrm{RP,O'Connor} \mathrm{MB}$. Control of the insect metamorphic transition by ecdysteroid production and secretion. Curr Opinion Insect Sci. 2021;43:11-20.

10. Liu HW, Wang LL, Tang X, Dong ZM, Guo PC, Zhao DC, Xia QY, Zhao P. Proteomic analysis of Bombyx mori molting fluid: insights into the molting. J Proteome. 2018:173:115-25.

11. Alexandratos A, Moulos P, Nellas I, Mavridis K, Dedos SG. Reassessing ecdysteroidogenic cells from the cell membrane receptors' perspective. Sci Rep. 2016;6:20229.

12. Hogenkamp DG, Arakane Y, Zimoch L, Merzendorfer H, Kramer KJ, Beeman RW, Kanost MR, Specht CA, Muthukrishnan S. Chitin synthase genes in Manduca sexta: characterization of a gut-specific transcript and differential tissue expression of alternately spliced mRNAs during development. Insect Biochem Mol Biol. 2005;35:529-40. 
13. Fukamizo T, Kramer KJ. Effect of 20-Hydroxyecdysone on Chitinase and Beta-N-Acetylglucosaminidase during the larval Pupal transformation of Manduca-Sexta (L). Insect Biochemistry. 1987;17:547-50.

14. Kramer KJ, Corpuz L, Choi HK, Muthukrishnan S. Sequence of a Cdna and expression of the gene encoding epidermal and gut Chitinases of Manduca Sexta. Insect Biochem Mol Biol. 1993;23:691-701.

15. Guo SY, Wu WM, Li SY, Liu Y, Ruan ZF, Ye MQ, Xiao Y, Zhong YJ, Cao Y, Li K, et al. 20-Hydroxyecdysone-upregulated proteases involved in Bombyx larval fat body destruction. Insect Mol Biol. 2018;27:724-38.

16. Yang $C$, Lin XW, Xu WH. Cathepsin L participates in the remodeling of the midgut through dissociation of midgut cells and activation of apoptosis via caspase-1. Insect Biochem Mol Biol. 2017:82:21-30.

17. Wright TR. The genetics of biogenic amine metabolism, sclerotization, and melanization in Drosophila melanogaster. Adv Genet. 1987;24:127-222.

18. Mackay TF, Heinsohn SL, Lyman RF, Moehring AJ, Morgan TJ, Rollmann SM. Genetics and genomics of Drosophila mating behavior. Proc Natl Acad Sci U S A. 2005;102:6622-9.

19. Di YQ, Han XL, Kang XL, Wang D, Chen CH, Wang JX, Zhao XF. Autophagy triggers CTSD (cathepsin D) maturation and localization inside cells to promote apoptosis. Autophagy. 2020. https://doi.org/10.1080/15548627. 2020.1752497

20. Ahmad T, Rajagopal R, Bhatnagar RK. Molecular characterization of chitinase from polyphagous pest Helicoverpa armigera. Biochem Biophys Res Commun. 2003;310:188-95.

21. Dong DJ, Liu PC, Wang JX, Zhao XF. The knockdown of ha-GRIM-19 by RNA interference induced programmed cell death. Amino Acids. 2012; 42:1297-307.

22. Hyun J, Hashimoto C. Physiological effects of manipulating the level of insulin-degrading enzyme in insulin-producing cells of Drosophila. Fly (Austin). 2011;5:53-7

23. Lin $X Y$, Smagghe $G$. Roles of the insulin signaling pathway in insect development and organ growth. Peptides. 2019;122:169923.

24. Mirth C, Truman JW, Riddiford LM. The role of the prothoracic gland in determining critical weight to metamorphosis in Drosophila melanogaster. Curr Biol. 2005;15:1796-807.

25. Pan J, Di YQ, Li YB, Chen CH, Wang JX, Zhao XF. Insulin and 20hydroxyecdysone oppose each other in the regulation of phosphoinositidedependent kinase-1 expression during insect pupation. J Biol Chem. 2018; 293:18613-23.

26. Zhao XF. Progress in understanding hormonal regulation during the postembryonic development of Helicoverpa armigera. J Integr Agric. 2020; 19:1417-28.

27. Vanderbliek AM, Meyers MB, Biedler JL, Hes E, Borst P. A 22-Kd protein (Sorcin/N19) encoded by an amplified gene in multidrug-resistant cells, is homologous to the calcium-binding light chain of Calpain. EMBO J. 1986;5: 3201-8.

28. Meyers MB, Fischer A, Sun YJ, Lopes CMB, Rohacs T, Nakamura TY, Zhou YY, Lee PC, Altschuld RA, McCune SA, et al. Sorcin regulates excitationcontraction coupling in the heart. J Biol Chem. 2003;278:28865-71.

29. Matsumoto T, Hisamatsu $Y$, Ohkusa T, Inoue N, Sato T, Suzuki S, Ikeda $Y$, Matsuzaki M. Sorcin interacts with sarcoplasmic reticulum $\mathrm{Ca}^{+}{ }^{-}$-ATPase and modulates excitation-contraction coupling in the heart. Basic Res Cardiol. 2005;100:250-62

30. Ilari A, Johnson KA, Nastopoulos V, Verzili D, Zamparelli C, Colotti G, Tsernoglou D, Chiancone E. The crystal structure of the sorcin calcium binding domain provides a model of $\mathrm{Ca}^{2+}$-dependent processes in the fulllength protein. J Mol Biol. 2002;317:447-58.

31. Liu W, Cai MJ, Zheng CC, Wang JX, Zhao XF. Phospholipase C gamma 1 connects the cell membrane pathway to the nuclear receptor pathway in insect steroid hormone signaling. J Biol Chem. 2014;289:13026-41.

32. Gupta K, Sirohi VK, Kumari S, Shukla V, Manohar M, Popli P, Dwivedi A. Sorcin is involved during embryo implantation via activating VEGF/PI3K/Akt pathway in mice. J Mol Endocrinol. 2018;60:119-32.

33. Sun Y, Liu W, Wang C, Meng Q, Liu Z, Huo X, Yang X, Sun P, Sun H, Ma X, Peng J, Liu K. Combination of dihydromyricetin and ondansetron strengthens antiproliferative efficiency of adriamycin in K562/ADR through downregulation of SORCIN: a new strategy of inhibiting P-glycoprotein. J Cell Physiol. 2019;234(4):3685-96.
34. Tuo H, Shu F, She S, Yang M, Zou XQ, Huang J, Hu HD, Hu P, Ren H, Peng $\mathrm{SF}$, et al. Sorcin induces gastric cancer cell migration and invasion contributing to STAT3 activation. Oncotarget. 2017;8:104258-71.

35. Hu YH, Li SJ, Yang M, Yan CH, Fan DM, Zhou Y, Zhang YJ, Yague E, Xiong DS. Sorcin silencing inhibits epithelial-to-mesenchymal transition and suppresses breast cancer metastasis in vivo. Breast Cancer Res Treat. 2014; 143:287-99.

36. Zhou JJ. Odorant-binding proteins in insects. Vitamins Hormones: Pheromones. 2010:83:241-72.

37. Steinbrecht RA. Odorant-binding proteins: expression and function. Ann N Y Acad Sci. 1998:855:323-32.

38. He P, Zhang J, Liu NY, Zhang YN, Yang K, Dong SL. Distinct expression profiles and different functions of odorant binding proteins in Nilaparvata lugens Stal. PLoS One. 2011;6:e28921.

39. Song HY, Dunbar JD, Zhang YX, Guo DQ, Donner DB. Identification of a protein with homology to Hsp90 that binds the Type-1 tumor-necrosisfactor receptor. J Biol Chem. 1995;270:3574-81.

40. Prodromou C, Roe SM, OBrien R, Ladbury JE, Piper PW, Pearl LH. Identification and structural characterization of the ATP/ADP-binding site in the Hsp90 molecular chaperone. Cell. 1997;90:65-75.

41. Liu W, Zhang FX, Cai MJ, Zhao WL, Li XR, Wang JX, Zhao XF. The hormonedependent function of Hsp90 in the crosstalk between 20-hydroxyecdysone and juvenile hormone signaling pathways in insects is determined by differential phosphorylation and protein interactions. Biochimica Et Biophysica Acta-General Subjects. 2013;1830:5184-92.

42. Jones $G$, Sarkari N. Basic isoforms of Hemolymph storage proteins expressed during larval metamorphosis. Arch Insect Biochem Physiol. 1993;24:55-64.

43. Jones G, Manczak M, Horn M. Hormonal regulation and properties of a new Group of Basic Hemolymph Proteins Expressed during insect metamorphosis. J Biol Chem. 1993;268:1284-91.

44. Zhao XF, Wang JX, Wang YC. Purification and characterization of a cysteine proteinase from eggs of the cotton boll worm, Helicoverpa armigera. Insect Biochem Mol Biol. 1998;28:259-64.

45. Kang XL, Zhang JY, Wang D, Zhao YM, Han XL, Wang JX, Zhao XF. The steroid hormone 20 -hydroxyecdysone binds to dopamine receptor to repress lepidopteran insect feeding and promote pupation. PLoS Genet. 2019;15:e1008331.

\section{Publisher's Note}

Springer Nature remains neutral with regard to jurisdictional claims in published maps and institutional affiliations.

Ready to submit your research? Choose BMC and benefit from:

- fast, convenient online submission

- thorough peer review by experienced researchers in your field

- rapid publication on acceptance

- support for research data, including large and complex data types

- gold Open Access which fosters wider collaboration and increased citations

- maximum visibility for your research: over $100 \mathrm{M}$ website views per year

At $\mathrm{BMC}$, research is always in progress.

Learn more biomedcentral.com/submission 